\title{
Internal Limiting Membrane Peeling and Dexamethasone Intravitreal Implant for the Management of a Retinal Macroaneurysm Rupture
}

\section{Pablo Sanjuán}

Centro de Oftalmología Barraquer: Centre d'Oftalmologia Barraquer

Víctor Lázaro-Rodríguez ( $\square$ victor.lazaro.bcn@gmail.com )

Centro de Oftamologia Barraquer https://orcid.org/0000-0003-3978-3594

Muhsen Samaan

Centro de Oftalmología Barraquer: Centre d'Oftalmologia Barraquer

Javier Elizalde

Centro de Oftalmología Barraquer: Centre d'Oftalmologia Barraquer

\section{Research Article}

Keywords: retinal arterial macroaneurysm, sub-ILM hemorrhage, pars plana vitrectomy, dexamethasone intravitreal implant injection

Posted Date: August 13th, 2021

DOI: https://doi.org/10.21203/rs.3.rs-278673/v1

License: (c) (i) This work is licensed under a Creative Commons Attribution 4.0 International License. Read Full License 


\section{Abstract}

Background: To describe an effective combined surgical and pharmacological approach for the management of a sub-internal limiting membrane (sub-ILM) macular hemorrhage and exudation related to a retinal arterial macroaneurysm (RAM) decompensation.

Methods: Pars plana vitrectomy (PPV) was performed, with an accurate forceps-assisted ILM removal, hemorrhage aspiration, and a dexamethasone intravitreal implant injection at the end of the surgery. Anatomical and functional outcomes were evaluated by fundus examination, spectral-domain optical coherence tomography (SD-OCT), optical coherence tomography angiography (OCT-A) and best-corrected visual acuity (BCVA).

Results: Postoperative outcome revealed that sub-ILM blood was successfully cleaned, and the RAM became progressively thrombosed with complete resolution of the exudation. Three months after surgery, BCVA improved from $20 / 400$ to $20 / 80$ with no signs of recurrence.

Conclusion: This combined surgical and pharmacological approach could be an effective technique for the management of sub-ILM hemorrhage secondary to RAM decompensation, and favors anatomical macular restoration and significant recovery of the visual acuity.

\section{Background}

Sub-internal limiting membrane (ILM) macular hemorrhages have been described in a variety of clinical settings ${ }^{1}$, the most common being Valsalva retinopathy and Terson's syndrome. However, a retinal arterial macroaneurysm (RAM) acute rupture, which usually presents with retinal and vitreous hemorrhages at different levels, may cause specific complications such as sub-ILM macular hemorrhage and consequently severe visual dysfunction.

Many therapeutic options ${ }^{1-5}$ have been proposed for the management of RAM complications including conservative and surgical procedures. We describe step-by-step a simple and effective combined technique to manage sub-ILM macular bleeding and intraretinal exudation secondary to a RAM decompensation.

\section{Case Presentation}

An 86-year-old man attended our office with complaints of a fifteen days history of sudden central blurred vision in his single right eye. Ocular examination revealed a BCVA of 20/400 related to a dome-shaped macular hemorrhage associated with a focal vascular lesion located in a main branch of the inferotemporal retinal artery, consistent with a RAM (Fig. 1A). Optical coherence tomography (Cirrus 5000, Zeiss, Germany) confirmed the localization of the hemorrhage below the ILM (Fig. 1B). OCT angiography (OCT-A) findings showed a high flow signal and clearly delineated the arterial focal dilation (Fig. 1C). 
Fluorescein and indocyanine green angiography revealed a gradually hyperfluorescent signal in the early stages corresponding to the RAM filling and a mild leakage in the latest phases of the angiogram.

Considering clinical symptoms and the central location of the hemorrhage in a single eye, a microincisional pars plana vitrectomy (PPV) was performed adding a dexamethasone intravitreal implant at the end of the procedure. SD-OCT examination at the immediate postoperative stage confirmed the disappearance of the sub-ILM hemorrhage. One month after surgery, an apparently partial RAM sclerosis with blood remnants were observed. Persistent hard exudates in a circinate pattern involving the macula appeared as intraretinal hyperreflective dots in the OCT. Three months after surgery, RAM sclerosis and reduction of the exudation was observed (Figs. 2A and 2C). OCT-A demonstrated a reduced flow signal through the RAM (Fig. 2B) and the BCVA improved to 20/80

\section{Methods}

A 23-gauge PPV was performed under peribulbar anesthesia and sedation. After completing the core and peripheral vitrectomy and checking that the posterior hyaloid was already detached, an ILM linear incision above the dome-shaped hemorrhage was done with a 25-gauge microvitreoretinal blade. Then, an ILM circular rhexis was performed with a $23 \mathrm{G}$ end-gripping forceps, and the exposed blood was gently aspirated with an active soft-tip extrusion cannula. In order to be sure that the ILM rhexis was complete, a Tano brush was used to check the borders of the dissection. Finally, a slow-release dexamethasone intravitreal implant was injected through the superotemporal sclerotomy using the Eckardt plate to keep the conjunctiva displaced and aligned with the scleral hole during this maneuver (see the edited surgical technique in the video file attached).

\section{Discussion And Conclusion}

A prompt microincisional PPV with ILM peeling is a simple and safe procedure that definitely accelerates visual acuity improvement in single eyes with central sub-ILM bleeding. Early evacuation of sub-ILM hemorrhage also prevents from developing preretinal fibrosis and contraction.

Other potential indications for this technique could include long-standing sub-ILM hemorrhages with incomplete spontaneous resorption, sub-ILM bleeding with suspicious subretinal component, and extensive sub-ILM hemorrhages with visual impairment.

Adjuvant intraocular steroids in a vitrectomized eye could also be useful to decrease RAM permeability, induce intraluminal thrombosis and accelerate edema and intraretinal exudates resolution.

To summarize, ILM-peeling and preretinal blood aspiration in association with dexamethasone intravitreal implant could be a safe and effective combined approach in patients with sub-ILM 
hemorrhage secondary to RAM acute decompensation, and favors anatomical restoration and significant visual recovery.

\section{Abbreviations}

BCVA: best-corrected visual acuity.

OCT-A: optical coherence tomography angiography.

PPV: pars plana vitrectomy.

RAM: retinal arterial macroaneurysm.

SD-OCT: spectral-domain optical coherence tomography.

Sub-ILM: sub-internal limiting membrane.

\section{Declarations}

\section{Funding}

This research did not receive any specific grant from funding agencies in the public, commercial, or notfor-profit sectors.

\section{Conflicts of interest}

The authors have no financial, proprietary or commercial interest in any materials presented herein.

\section{Ethics approval}

Not applicable

\section{Consent to participate}

The authors obtained written informed consent to participate from the patient.

\section{Consent for publication}

A declaration of consent has been obtained from the patient to publish the attached images. 


\section{Availability of data and material}

Not applicable.

\section{Code availability.}

Not applicable

\section{Authors' contribution}

All authors have contributed and approved the final manuscript.

\section{References}

1. De Maeyer K, Van Ginderdeuren R, Postelmans L, Stalmans P, Van Calster J. Sub-inner limiting membrane haemorrhage: causes and treatment with vitrectomy. Br J Ophthalmol. 2007;91:869-72.

2. Hotta K, Hotta J. Case of recurrent macular haemorrhage after removal of a sub-internal limiting membrane haematoma secondary to retinal artery macroaneurysm. Clin Exp Ophthalmol. 2006;34:610-12.

3. Zhao P, Hayashi H, Oshima K, Nakagawa N, Ohsato M. Vitrectomy for macular hemorrhage associated with retinal arterial macroaneurysm. Ophthalmology. 2000;107(3):613-7.

4. Cahuzac A, Scemama C, Mauget-Faysse M, Sahel J-A, Wolff B. Retinal arterial macroaneurysms: clinical, angiographic, and tomographic description and therapeutic management of a series of 14 cases. Eur J Ophthalmol 2016 Jan-Feb; 26(1): 36-43.

5. Koinzer S, Heckmann J, Tode J, Roider J. Long-term, therapy-related visual outcome of 49 cases with retinal arterial macroaneurysm: a case series and literature review. $\mathrm{Br} \mathrm{J}$ Ophthalmol. 2015;99(10):1345-53.

\section{Figures}




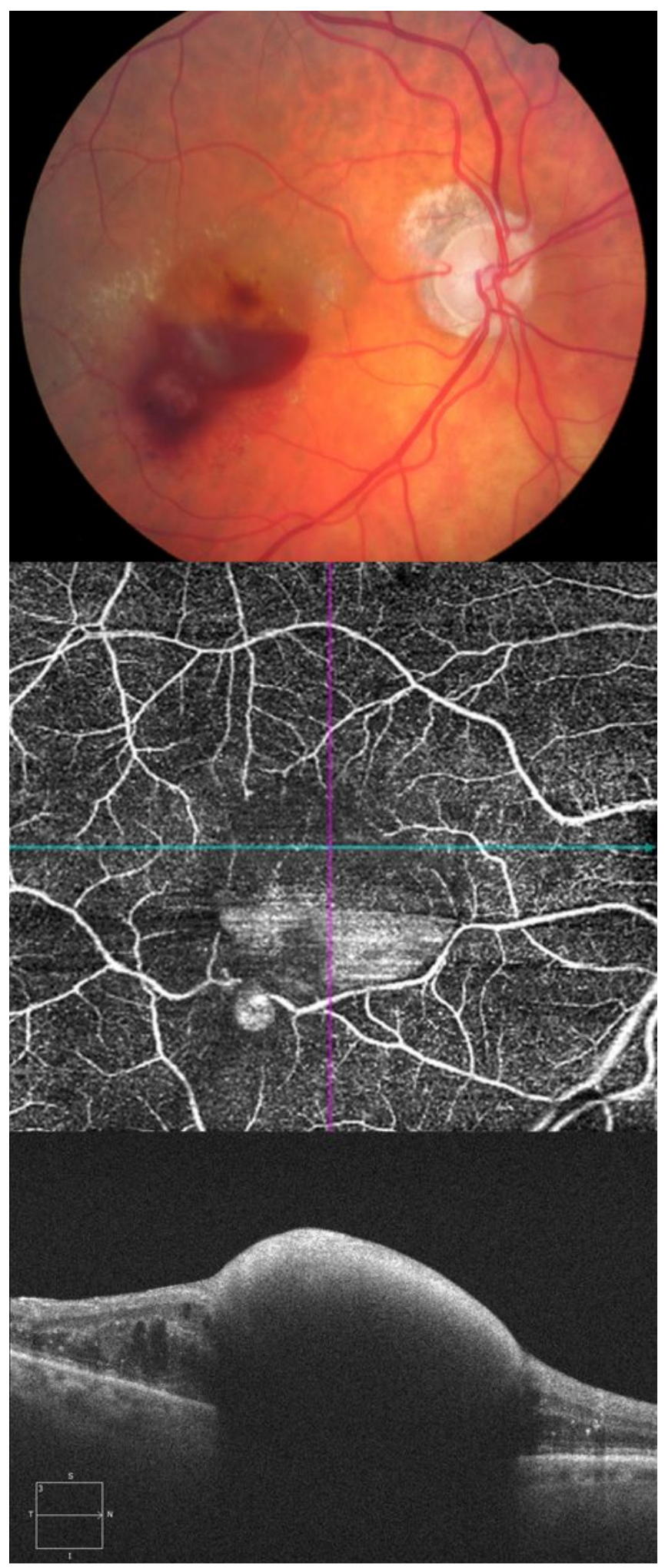

\section{Figure 1}

Preoperative fundus photograph shows a sub-internal limiting membrane (ILM) macular hemorrhage and a retinal arterial macroaneurysm (RAM) along the inferotemporal retinal artery (A). OCT-angiography (OCT-A) demonstrates high flow signal through the RAM (B). Optical coherence tomography (OCT) at presentation reveals an inner retinal layer hyperreflectivity and outer retinal layer shadowing consistent with the sub-ILM hemorrhage (C). 


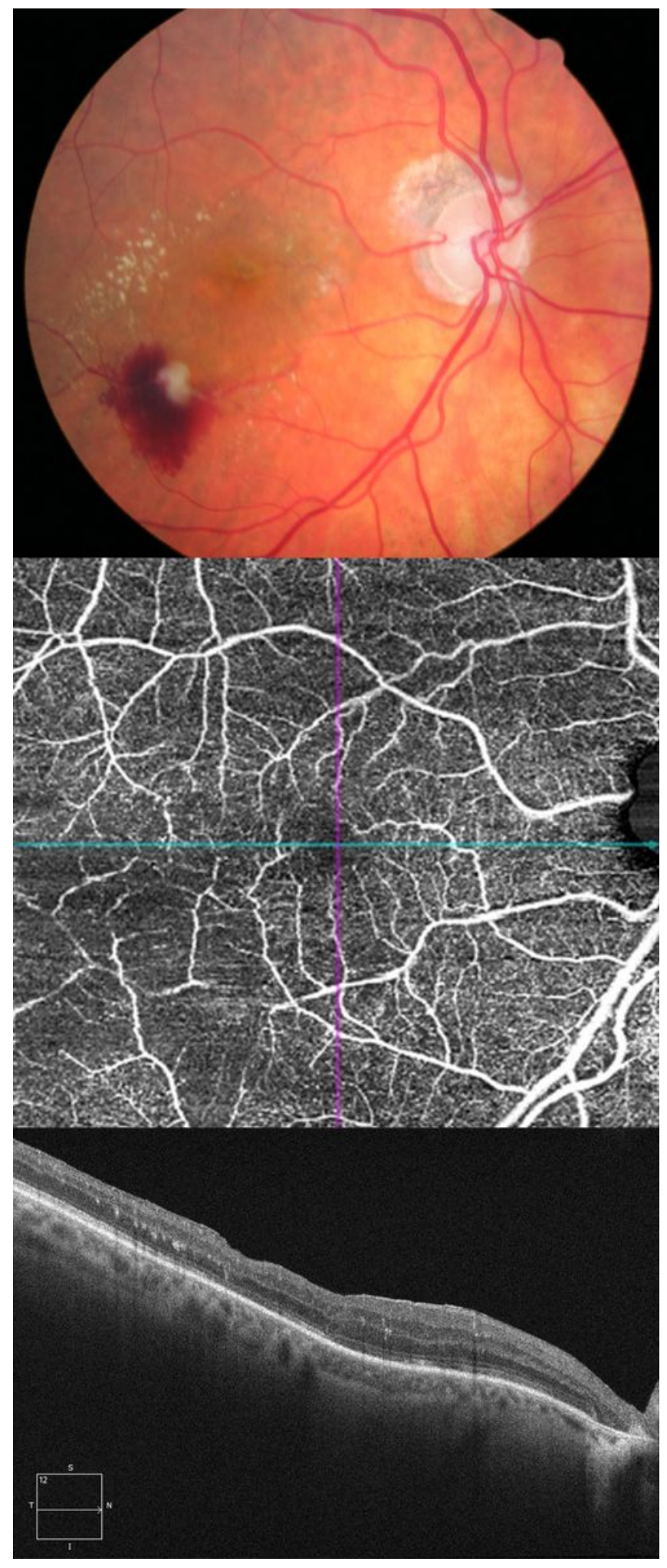

Figure 2

Three months postoperative fundus photograph shows RAM sclerosis with hard exudates in a circinate pattern involving the macula (A). OCT-A reveals reduced flow signal through the RAM (B). OCT demonstrates recovery of the integrity of the retinal layers $(C)$.

\section{Supplementary Files}


This is a list of supplementary files associated with this preprint. Click to download.

- 603110.mp4 